\title{
Nonhydrostatic compression of elastically anisotropic polycrystals. II. Direct compression and plastic deformation
}

\author{
J. W. Otto and J. K. Vassiliou \\ Department of Physics, Villanova University, Villanova, Pennsylvania 19085 \\ G. Frommeyer \\ Max-Planck-Institut für Eisenforschung, Max-Planck-Strasse 1, D-40237 Düsseldorf, Germany
}

(Received 7 July 1997)

\begin{abstract}
The effects of nonhydrostatic conditions on the compression behavior of elastically anisotropic polycrystals have been studied by energy-dispersive x-ray diffraction of a foil of $\mathrm{Cu}_{3} \mathrm{Au}$ compressed directly between diamond anvils. A volume expansion between 0.3 and $1 \mathrm{GPa}$ is explained to result from the introduction of dislocations. A volume incompressibility accompanied by saturation in stress, strain, and the stacking fault probability between 1 and $2 \mathrm{GPa}$ is due to work hardening. A complete pressure cycle, including repressurization after pressure release, and the compression of $\mathrm{Cu}_{3} \mathrm{Au}$ previously deformed by filing demonstrate that mutual repulsion between lattice defects also contributes to the volume incompressibility. The pressure region of incompressibility terminates at a discontinuity which occurs when the yield strength of the plastically deformed material is exceeded. The compression above this point is elastic and isotropic. The yield strength is estimated from the offset of the measured compression curves from the hydrostat. [S0163-1829(98)01006-6]
\end{abstract}

\section{INTRODUCTION}

The compression behavior of a polycrystalline elastically anisotropic material under increasing nonhydrostatic conditions has been detailed in previous studies using various pressure media. ${ }^{1,2}$ A region of elastic incompressibility was explained to result from work hardening. A discontinuity in the compressibility occurs when elastic compression again sets in above the yield point. Because of the use of liquid or solid pressure transmitting media in these studies, the yield point and the region of elastic incompressibility occurred at relatively high values of hydrostatic to uniaxial stress (this is related to the shear strength of the pressure-transmitting medium relative to that of the sample). ${ }^{1,2}$ In order to gain more insight into the physical processes during compression under nonhydrostatic conditions, a foil of $\mathrm{Cu}_{3} \mathrm{Au}$ was directly compressed between two diamonds. In this case, the uniaxial stress component is increased relative to the hydrostatic component at low pressures compared with the previous studies. ${ }^{1,2}$ This setup allows for direct comparison with shock-wave studies in which a sample is deformed (compressed) at very high rates of strain and variable temperature without a pressure medium or gasket. The compression of a foil deformed in a first pressure cycle is compared with the compression of a foil previously deformed by filing at liquid nitrogen temperatures. The results are discussed with respect to earlier studies of the foil in various pressure media.,

\section{EXPERIMENTAL AND ANALYTICAL PROCEDURES}

The choice of sample and experimental parameters has been described in detail before. ${ }^{1,2}$ The scattering angles of the energy-dispersive $\mathrm{x}$-ray-diffraction experiments were $2 \theta=10.66^{\circ}$ for the experiment using filings and $2 \theta$ $=10.60^{\circ}$ and $2 \theta=10.72^{\circ}$ for two experiments compressing the foil directly between the diamond anvils without a pres- sure medium or gasket. In the direct compression experiments, single flakes of $\mathrm{Cu}_{3} \mathrm{Au}$ were used. Compression to 6.7 $\mathrm{GPa}(2.8 \mathrm{GPa}$ on second compression) and 5.9 $\mathrm{GPa}$ resulted in reductions in thickness from $55 \mu \mathrm{m}$ to $17 \mu \mathrm{m}$ and from $50 \mu \mathrm{m}$ to $14 \mu \mathrm{m}$, respectively. In order to obtain a fully plastically deformed starting material for another experiment, a foil was filed under liquid nitrogen. The foil remained ductile at these temperatures. For pressure measurement by the fluorescence method, ${ }^{3,4}$ a single grain of ruby was attached to the piston diamond (in the case of filings in paraffin oil, vacuum grease was used as the gluing agent). The ruby linewidths in the direct compression experiments remained close to the value at ambient conditions. This is not surprising considering the difference in strength between $\mathrm{Cu}_{3} \mathrm{Au}$ and $\mathrm{Al}_{2} \mathrm{O}_{3}$. The shear modulus of $\mathrm{Cu}_{3} \mathrm{Au}$ at $1 \mathrm{GPa}$ is comparable to that of $\mathrm{NaCl}^{1}$

Exposure times of the energy-dispersive x-ray-diffraction experiments at beam line F3 at HASYLAB (Refs. 5 and 6) were chosen such that sufficient counts for a profile analysis of the reflections up to (311) were accumulated. This meant exposure times of about 140 min with dead times of $0.8-$ $1 \%$. Total counts in (111) were of the order of 40000 for the direct compression experiment and 20000-30000 for the experiment on filings. In a second direct compression experiment to check the reproducibility of the results, exposure times were shorter in order to also allow for pressure scans to be carried out.

\section{RESULTS}

\section{A. Direct compression of an undeformed foil between diamonds}

The pressure dependence of the scaled volume is illustrated with the (111) and (200) lattice spacings which represent the lowest and highest values observed as deformation 


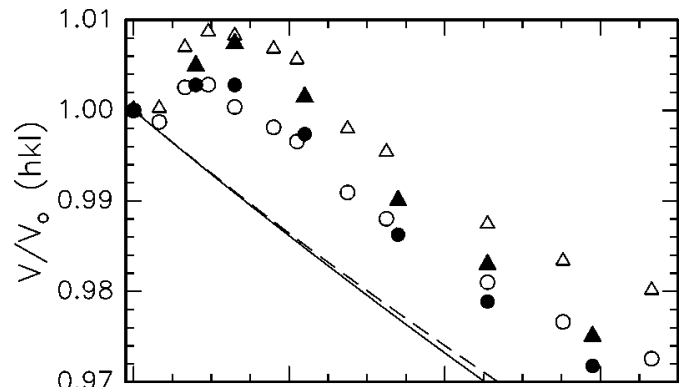

(a)

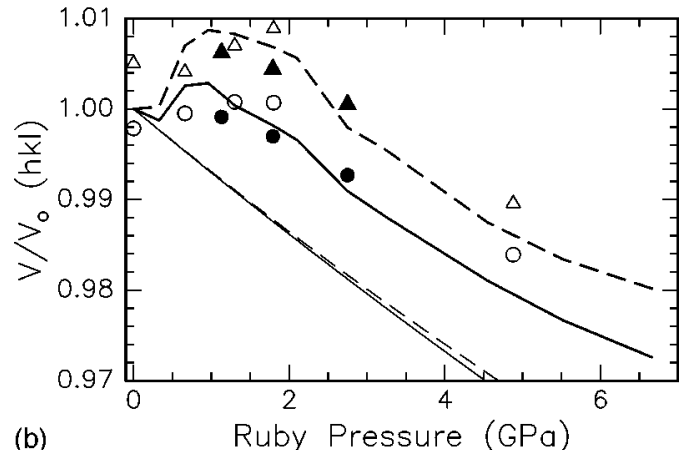

(b)

Ruby Pressure (GPa)

FIG. 1. The scaled volume for directly compressed $\mathrm{Cu}_{3} \mathrm{Au}$ foil calculated from the (111) and (200) lattice spacings (circles and triangles, respectively) vs pressure. The data for the first pressurization are given in the top part (a) with open and solid symbols denoting two different experiments (Tables I and II). The open and solid symbols in the bottom part (b) represent the data for pressure release and second pressurization, respectively. The heavy solid and dashed lines in the bottom part connect the data points of the first experiment shown in (a). The scaled volumes calculated from the (111), (220), and (222) lattice spacings coincide (see Table I). The scaled volumes calculated from the (311) spacing lie in between those calculated from (111) and (200). Light solid and dashed lines are first- and second-order Birch-Murnaghan equations of state with $B_{0}=139.6$ and $B_{0}=140.6$, respectively, as explained in Ref. 2 .

sets in [Figs. 1(a) and 1(b)]. The full lists of $d$ spacings are given in Tables I and II. On first compression, the volume remains essentially unchanged to $0.3 \mathrm{GPa}$ [except for a slight decrease in (111) and (222)]. There is an increase in volume starting between 0.3 and $0.7 \mathrm{GPa}$ and continuing up to $1 \mathrm{GPa}$ measured from all lattice spacings except for (200) and (311) of the second experiment which continue to increase to 1.3 $\mathrm{GPa}$. The volume calculated from the (200) lattice spacing increases more strongly than that calculated from the other reflections. The increase is about $0.5 \%$ for (111) and $0.9 \%$ for (200). At pressures above a discontinuity in the compressibility between 1 and $1.3 \mathrm{GPa}$, the compression behavior is smooth to the highest pressure investigated. The compressibility above $2.2 \mathrm{GPa}$ measured in the second compression experiment is higher than that measured in the first one. Note that the difference in volumes calculated from the (200) and the other reflections increases to $2 \mathrm{GPa}(1.3$ GPa during the second experiment) and decreases slightly above that pressure. On pressure release [Fig. 1(b)], the volume strain is $+0.5 \%$ in (200) and $-0.3 \%$ in (111) at ambient conditions; there is some relaxation for the other lattice spacings. On second compression [Fig. 1(b)], the volume remains essentially unchanged to $1.3 \mathrm{GPa}$. Above $1.3 \mathrm{GPa}$,
TABLE I. $d$ spacings as a function of pressure for the direct compression of $\mathrm{Cu}_{3} \mathrm{Au}$ foil between diamond anvils at an angle of $2 \theta=10.72^{\circ}$.

\begin{tabular}{cccccc}
\hline \hline $\begin{array}{c}h k l \\
\text { Pressure }(\mathrm{GPa})\end{array}$ & 111 & 200 & $\begin{array}{c}220 \\
\text { spacings }(\AA)\end{array}$ & 311 & 222 \\
\hline 0.0001 & 2.168 & 1.876 & 1.327 & 1.132 & 1.084 \\
0.33 & 2.167 & 1.876 & 1.327 & 1.132 & 1.083 \\
0.66 & 2.170 & 1.880 & 1.330 & 1.134 & 1.085 \\
0.96 & 2.170 & 1.881 & 1.330 & 1.135 & 1.085 \\
1.30 & 2.168 & 1.881 & 1.329 & 1.134 & 1.084 \\
1.80 & 2.166 & 1.880 & 1.326 & 1.133 & 1.083 \\
2.10 & 2.165 & 1.879 & 1.326 & 1.133 & 1.083 \\
2.75 & 2.161 & 1.875 & 1.324 & 1.130 & 1.081 \\
3.25 & 2.159 & 1.873 & 1.322 & 1.129 & 1.080 \\
4.55 & 2.154 & 1.868 & 1.319 & 1.126 & 1.077 \\
5.52 & 2.151 & 1.865 & 1.317 & 1.124 & 1.076 \\
6.66 & 2.148 & 1.863 & 1.315 & 1.123 & 1.074 \\
& & & & & \\
4.88 & 2.156 & 1.869 & 1.320 & 1.127 & 1.078 \\
1.80 & 2.168 & 1.881 & 1.328 & 1.134 & 1.084 \\
1.30 & 2.168 & 1.880 & 1.328 & 1.133 & 1.084 \\
0.66 & 2.167 & 1.878 & 1.328 & 1.133 & 1.084 \\
0.0001 & 2.166 & 1.879 & 1.328 & 1.132 & 1.083 \\
1.13 & 2.167 & 1.880 & 1.327 & 1.133 & 1.084 \\
1.79 & 2.166 & 1.879 & 1.326 & 1.132 & 1.083 \\
2.75 & 2.162 & 1.876 & 1.325 & 1.131 & 1.081 \\
\hline \hline & & & & & \\
\hline
\end{tabular}

the volume data of the second compression closely reproduce those of the first one in this pressure range.

The compressive stress increases immediately on first pressurization and saturates at a pressure of around $1.3 \mathrm{GPa}$ in the two experiments (Fig. 2). The two values around 2 $\mathrm{GPa}$ in the first experiment are higher than the values above this pressure; this fact and the trend of the data of the second run suggest that the values at $2 \mathrm{GPa}$ in the first experiment are too high. There is no stress relaxation on pressure release and no increase in stress on second compression to $3 \mathrm{GPa}$. The agreement between the two experiments in the trends of the stresses with pressure is good, while the stresses calculated for the second experiment are lower by $0.1-0.2 \mathrm{GPa}$.

The stacking fault probability calculated from the relative separation of the (111) and (200) reflections increases from

TABLE II. $d$ spacings as a function of pressure for the direct compression of $\mathrm{Cu}_{3} \mathrm{Au}$ foil between diamond anvils at an angle of $2 \theta=10.6^{\circ}$.

\begin{tabular}{lccccc}
\hline \hline $\begin{array}{c}h k l \\
\text { Pressure }(\mathrm{GPa})\end{array}$ & 111 & 200 & $\begin{array}{c}220 \\
\text { spacings }(\AA)\end{array}$ & 311 & 222 \\
\hline 0.0001 & 2.168 & 1.878 & 1.328 & 1.132 & 1.084 \\
0.8 & 2.170 & 1.881 & 1.330 & 1.134 & 1.085 \\
1.3 & 2.170 & 1.882 & 1.330 & 1.135 & 1.085 \\
2.2 & 2.166 & 1.879 & 1.327 & 1.132 & 1.083 \\
3.4 & 2.158 & 1.872 & 1.323 & 1.129 & 1.079 \\
4.55 & 2.153 & 1.867 & 1.319 & 1.126 & 1.077 \\
5.9 & 2.148 & 1.862 & 1.316 & 1.123 & 1.074 \\
\hline \hline
\end{tabular}




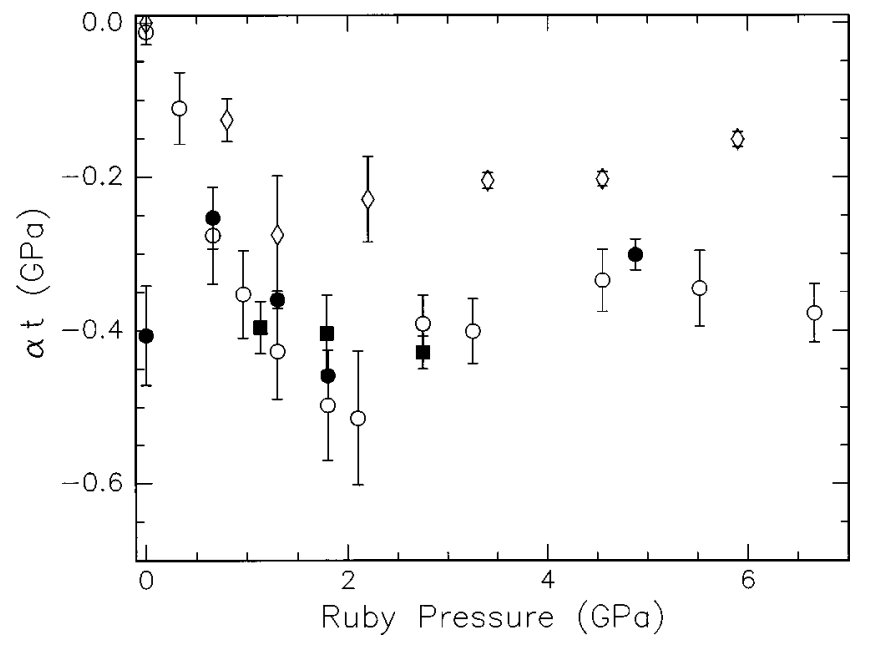

FIG. 2. The product $\alpha t$ (fraction of Reuss state in the sample times the stress) for $\mathrm{Cu}_{3} \mathrm{Au}$ with increasing confining pressure (open symbols), pressure release (solid circles), and second pressurization (solid squares). The open rhombs are for a second compression experiment. Note the residual compressive stress of $0.4 \mathrm{GPa}$. The error bars are from the fit to the total strain versus lattice direction (Ref. 1).

ambient conditions to $1.3 \mathrm{GPa}$ (Fig. 3). The values above 2 $\mathrm{GPa}$ in the first experiment and comparison with the values of the second experiment suggest that the values between 1 and $2 \mathrm{GPa}$ in the first experiment are too high. While the trends agree, the values of the second experiment are lower than those of the first experiment. On pressure release, there is strong scatter in the values between 0.023 (at ambient conditions) and 0.011 . On second pressurization, the stacking fault probability remains constant at around 0.018 .

The trend with pressure of the Voigt fit parameters for the reflections (111) through (311) for the first run is illustrated in Figs. 4(a)-4(d) with (from top to bottom) the full widths at half maximum (FWHM) (raw data) and the corrected Lorentzian fraction $\beta_{C} / \beta$ and the corrected Gaussian and Lorentzian integral widths. The FWHM of these reflections

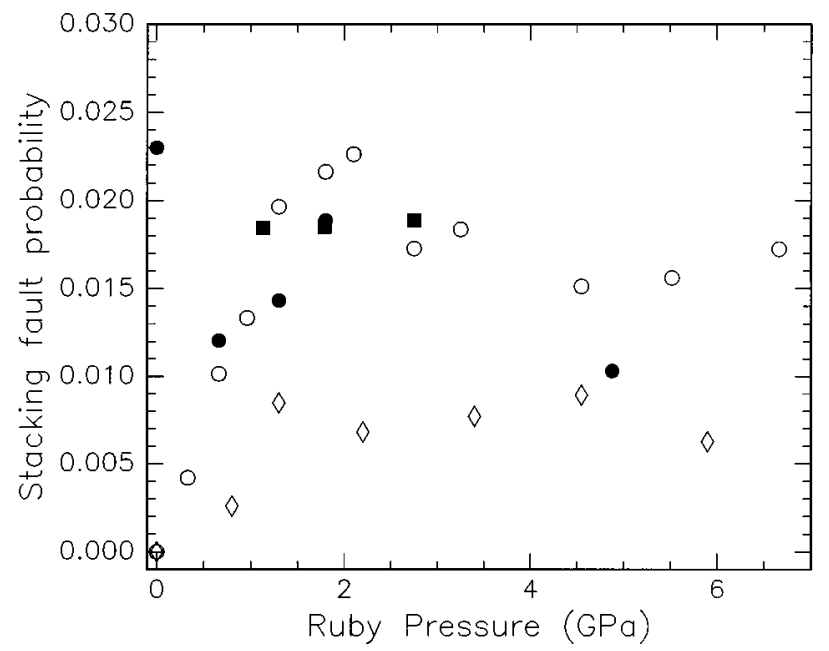

FIG. 3. The stacking fault probability in $\mathrm{Cu}_{3} \mathrm{Au}$ calculated from the relative shift in the (111) and (200) spacings. Note the saturation at about $1.3 \mathrm{GPa}$ (i.e., around the discontinuity in the compressibility). Symbols as for Fig. 2. for the second run are shown in Fig. 5; in this experiment, a profile analysis could be carried out only for the (111) and (200) reflections because of insufficient counts in the other reflections. The trends in the above parameters are similar for the observed reflections and are most clearly exhibited by the (200) reflection in the first run [Fig. 4(b)]. On first application of pressure to $0.3 \mathrm{GPa}$, there is an increase in all parameters. They remain constant to $1 \mathrm{GPa}$ [Fig. 4(b)] followed by a jump between 1 and $1.3 \mathrm{GPa}(0.8$ and $1.3 \mathrm{GPa}$ in the second experiment, Fig. 5). The parameters continue to increase, with most or all of the increase taking place up to 2 GPa. The Lorentzian integral width and Lorentzian fraction in the first experiment saturate at about $4 \mathrm{GPa}$, while the behavior was found to be no different from that of the Gaussian integral width in the second experiment. Above about 2 $\mathrm{GPa}$, Gaussian and Lorentzian integral widths are essentially equal in the first experiment with $\beta_{C} / \beta$ saturating at a value around 0.6 (0.5 in the second experiment). Note the excellent agreement in the FWHM for the two experiments [except for the broader (311) in the first experiment].

On pressure reduction, there is little change in $\beta_{C} / \beta$ while $\beta_{C}$ relaxes by around $15 \%$ [except for (111) where there is no relaxation] and $\beta_{G}$ by $20-30 \%$ [except in (111) where the relaxation is about $15 \%$ ] mostly below $2 \mathrm{GPa}$. On second compression, the parameters mostly remain constant or increase slightly with values similar to those on first compression. The following exceptions to the above general trends were noted in the first experiment: (i) $\beta_{C} / \beta$ for (200) does not increase at $0.3 \mathrm{GPa}$ on first compression because $\beta_{C}$ and $\beta_{G}$ increase by the same relative amounts. (ii) The FWHM of the (111) and (311) reflections continue to increase between 2 and $4 \mathrm{GPa}$. (iii) The initial value of $\beta_{G}$ for (111) appears to be too high so that the increase to $0.3 \mathrm{GPa}$ is masked. No change in $\beta_{C} / \beta$ is seen above $1 \mathrm{GPa}$, and $\beta_{C} \leqslant \beta_{G}$. There appears to be relaxation in the FWHM and $\beta_{G}$ immediately on first pressure reduction.

The strong scatter in $\beta_{C} / \beta$ for (220) [Fig. 4(c)] illustrates the general problems of a possible anticorrelation of $\beta_{C}$ and $\beta_{G}$ [note especially the seeming increase in $\beta_{C} / \beta$ of (311) between 0.3 and $1 \mathrm{GPa}$, Fig. 4(d)], of the overlap of reflections [in this case with the escape peak of (311)] and of insufficient counting statistics.

The trends in effective crystallite size and the strain follow the Lorentzian and Gaussian integral widths from which they are calculated ${ }^{2}$ and hence no graphs are shown. Values for the effective size and strain for ambient conditions before and after, and for the maximum pressure reached during, the first pressure cycle are given in Table III for the first experiment. The broadening expected from the calculated stacking fault probability is larger than the observed Lorentzian widths. This may mean that the stacking fault probability is less than calculated or that the size effect also has a Gaussian component. The strain calculated from the Gaussian broadening roughly doubles on application of the maximum pressure; about half of this increase is recovered on pressure release.

Two scans in the (horizontal) diffraction plane across the tips of the diamond anvils were carried out to investigate the deformation. At $2.2 \mathrm{GPa}$ (just above the discontinuity in the compressibility), the deformation as judged from the relative separations of the lattice spacings [Fig. 6(a), top, and Table 

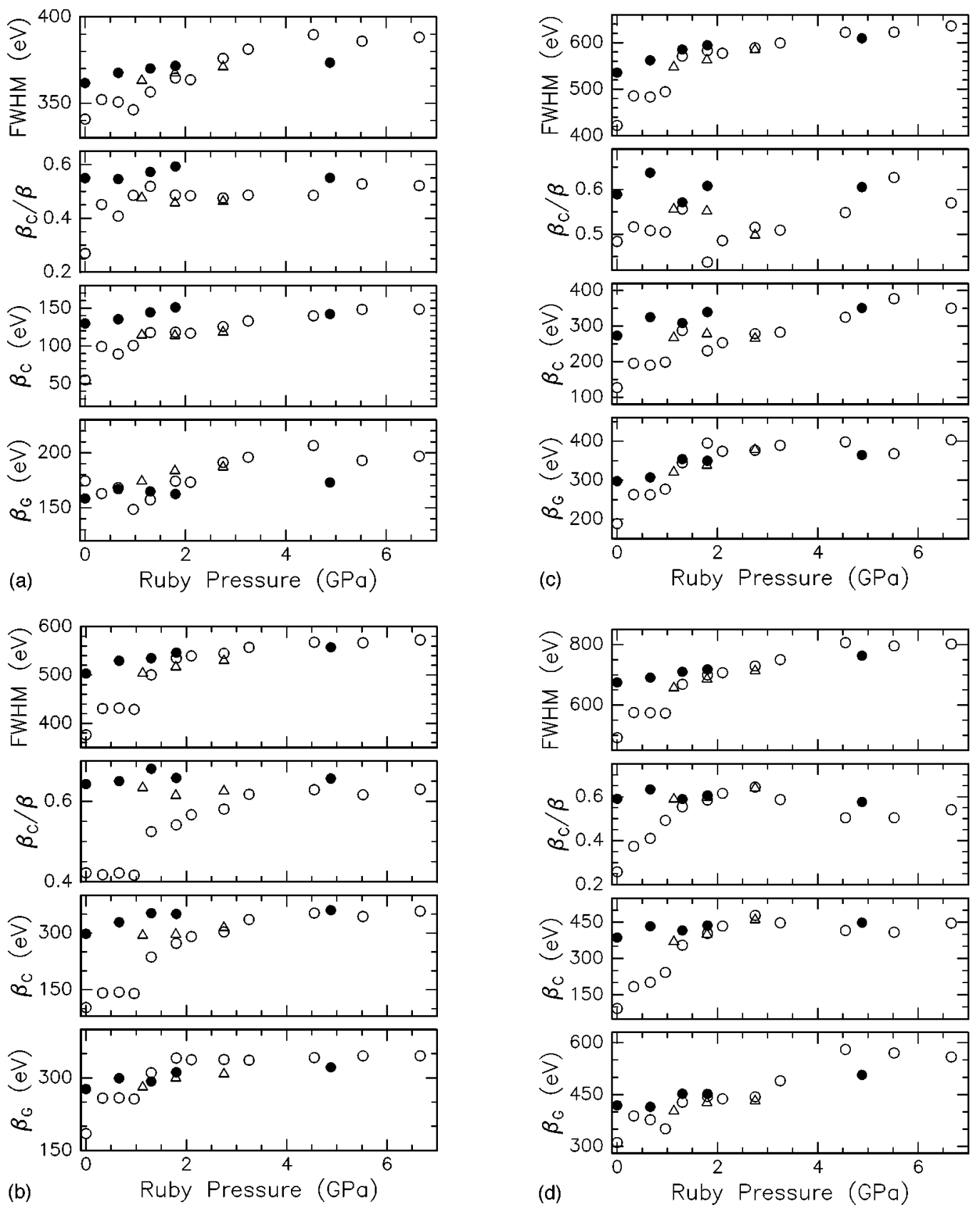

FIG. 4. Voigt fit parameters to the reflections (111) (a), (200) (b), (220) (c), and (311) (d). From top to bottom, the parameters are the FWHM, the Lorentzian fraction $\beta_{C} / \beta$, and the Lorentzian and Gaussian integral widths. Open circles are for first pressurization, solid circles for pressure release, and open triangles for second compression.

IV], the stresses [Fig. 6(a), bottom] and from the line profiles (not shown) is quite homogeneous: The foil even at the outer edges of the diamond anvils is deformed. As expected from the compression behavior at the center as a function of pressure, the volume of the foil is expanded relative to the volume at ambient conditions and there is a compression component towards the center of the anvils [Fig. 6(a), top]. At $5.9 \mathrm{GPa}$ [Table IV and Fig. 6(b)], there is deformation with apparently no compression only near the outer edges of the anvils. It is not clear whether the reduced separation of (111) and (200) at 5.9 GPa compared with that at $2.2 \mathrm{GPa}$ is real or whether it does represent a reduction in the stacking fault probability with pressure. There was a large amount of scatter in the line profiles in the scan at $5.9 \mathrm{GPa}$ due to insuffi- cient statistics. While line profiles were much broader than the starting value (as expected already from the scan at 2.2 GPa), an apparent increase in the FWHM towards the center was found only for (111), (200), and (311). The stresses in the foil appear to be constant across the tips of the diamond anvils [Fig. 6(b), bottom].

A low-energy tail was present in the (111) reflection and between 0.3 and about $1.3 \mathrm{GPa}$ in the (200) reflection of the first experiment (Fig. 7). This tail is probably not due to electronic effects in the detector (such as insufficient charge collection at high count rates) since the dead time was always below $0.9 \%$ and the count rate in (111) was below 10 counts/s. The maximum intensity of the tailing is $0.1 \%$ of the peak intensity. It was noted also in some of the other experi- 


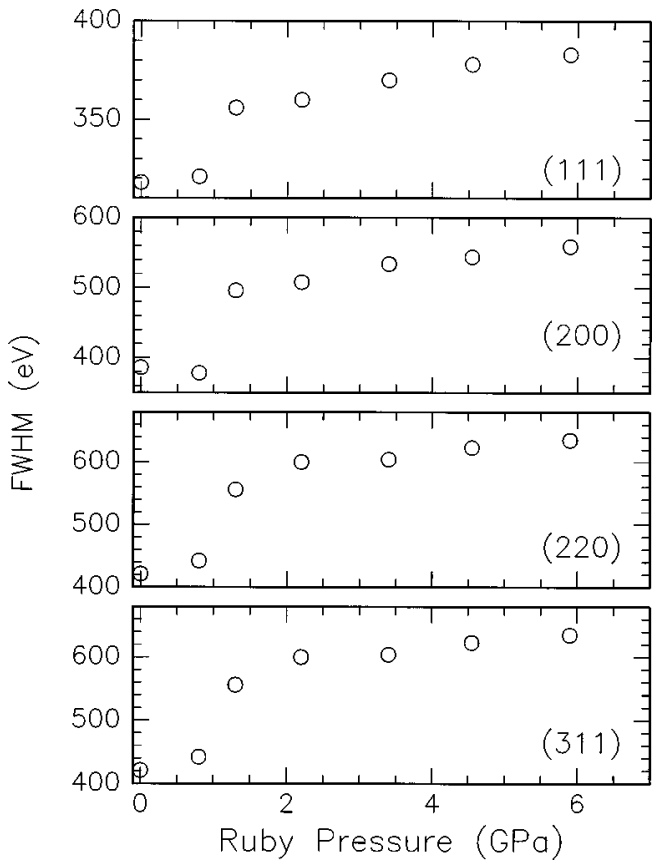

FIG. 5. FWHM for the (111) through (311) reflections in the second run. Note the excellent agreement with the data shown in Fig. 4 [except for the large FWHM for (311) in the first run].

ments with $\mathrm{a} \mathrm{Cu}_{3} \mathrm{Au}$ foil. The origin of the tailing is unclear at present but may indicate the presence of line defects other than $\{111\}$ stacking faults. Dislocations on $\{001\}$ planes are also known in $\mathrm{Cu}_{3} \mathrm{Au}{ }^{7}$

\section{B. Compression of filings in paraffin oil}

Filings of $\mathrm{Cu}_{3} \mathrm{Au}$ made at liquid nitrogen temperatures were compressed to $5 \mathrm{GPa}$. The lattice spacing of (200) increases on filing (Table V). The stacking fault probability calculated from the separation of the (111) and (200) centroids is $1 / 51$ (the centroids and not the peak maxima were used because of the strongly varying background underneath the peaks). This agrees well with a measurement of another sample at $2 \theta=14.922^{\circ}(1 / 50)^{4}$ and with a determination by
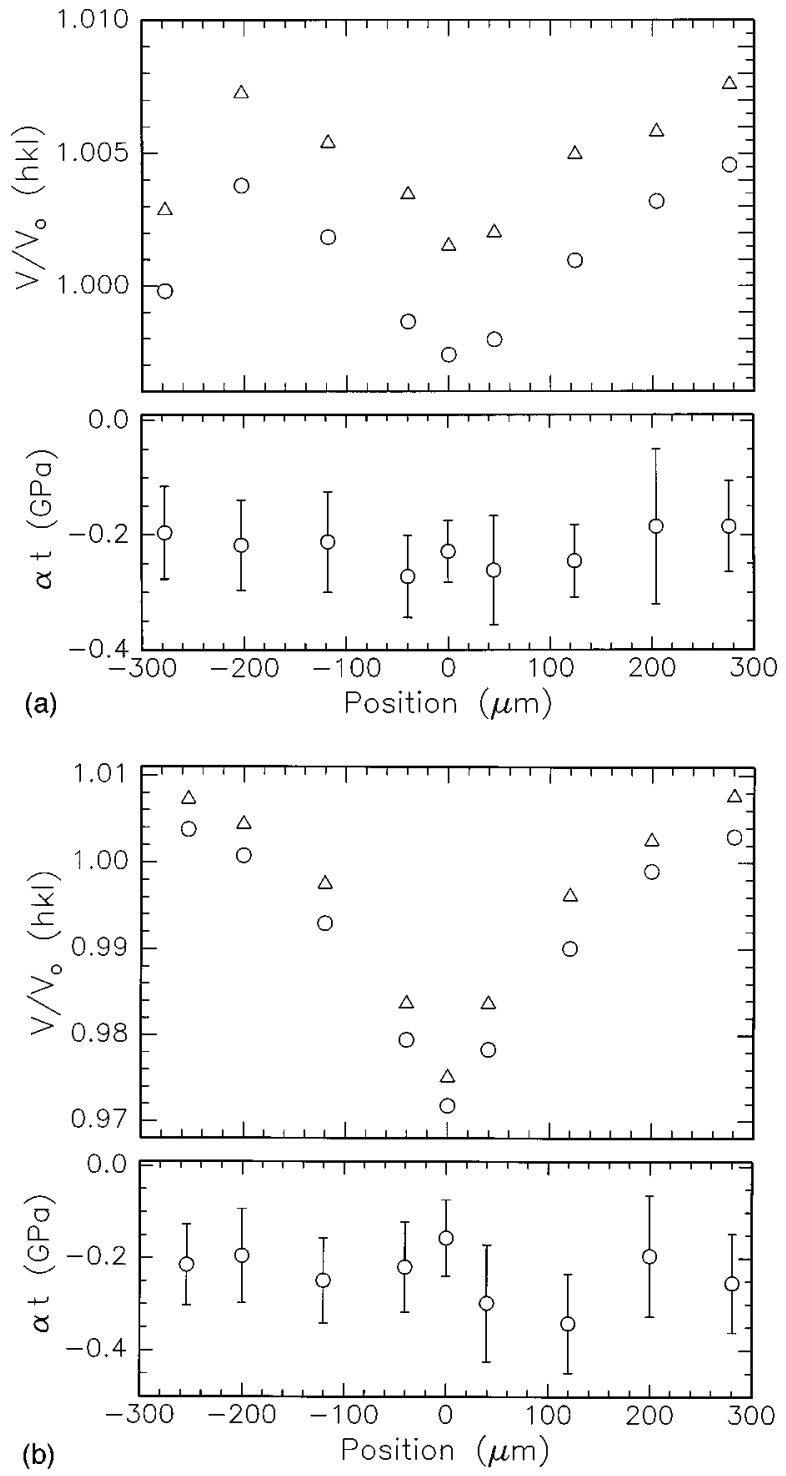

FIG. 6. Scaled volumes calculated from the (111) and (200) lattice spacings (open circles and triangles, respectively, top) and stresses (bottom) for radial scans across the tips of the diamond anvils at $2.2 \mathrm{GPa}(\mathrm{a})$ and $5.9 \mathrm{GPa}(\mathrm{b})$.

TABLE III. Effective crystallite size, true crystallite size, and strain $(\epsilon)$ for the directly compressed foil (at ambient conditions before and after the run and at the maximum pressure of $6.7 \mathrm{GPa}$ ) and for the foil deformed by filing (before and after filing, at $4.8 \mathrm{GPa}$ and after the pressure cycle at ambient conditions). The effective size was calculated from $\beta_{C}$, the true size from $\beta_{C}$ after subtracting the particle size effect of stacking faults. The strain $\epsilon$ was calculated from $\beta_{G}$ [see Eqs. (7) and (8) of Ref. 2]. No data are shown for the (311) reflection at ambient conditions before filing because of overlap with the tungsten fluorescence (from the collimators and slits).

\begin{tabular}{|c|c|c|c|c|c|c|c|c|c|c|c|c|c|}
\hline & \multirow{2}{*}{$\begin{array}{c}h k l \\
\text { Pressure (GPa) }\end{array}$} & 111 & 200 & 220 & 311 & 111 & 200 & 220 & 311 & 111 & 200 & 220 & 311 \\
\hline & & \multicolumn{4}{|c|}{ Effective size (nm) } & \multicolumn{4}{|c|}{ True size $(\mathrm{nm})$} & \multicolumn{4}{|c|}{ Strain $\left(10^{-4}\right)$} \\
\hline \multirow{4}{*}{$\begin{array}{l}\text { Direct } \\
\text { compression }\end{array}$} & 0.0001 & 121 & 65 & 52 & 71 & 121 & 65 & 52 & 71 & 29 & 26 & 19 & 27 \\
\hline & 6.7 & 45 & 19 & 19 & 15 & & & & & 32 & 49 & 40 & 47 \\
\hline & 0.0001 & 51 & 22 & 24 & 17 & & & & & 26 & 39 & 30 & 36 \\
\hline & before & 333 & 100 & 82 & & 333 & 100 & 82 & & 21 & 25 & 21 & \\
\hline \multirow{3}{*}{ Filings } & after & 17 & 9 & 8 & 6 & 40 & 26 & 15 & 8 & 33 & 60 & 39 & 56 \\
\hline & 4.8 & 14 & 8 & 8 & 7 & 24 & 19 & 15 & 9 & 32 & 70 & 35 & 35 \\
\hline & 0.0001 & 17 & 10 & 8 & 6 & 28 & 36 & 14 & 8 & 34 & 71 & 35 & 38 \\
\hline
\end{tabular}


TABLE IV. $d$ spacings of $\mathrm{Cu}_{3} \mathrm{Au}$ directly compressed between diamond anvils at pressures of 2.2 and 5.9 $\mathrm{GPa}$ as a function of distance from the center of the anvils.

\begin{tabular}{|c|c|c|c|c|c|c|c|c|c|c|}
\hline & \multirow[t]{2}{*}{$h k l$} & \multicolumn{9}{|c|}{ Position $(\mu \mathrm{m})$} \\
\hline & & -278 & -203 & -118 & -40 & 0 & 45 & 124 & 204 & 276 \\
\hline \multirow{7}{*}{$2.2 \mathrm{GPa}$} & 111 & 2.168 & 2.171 & 2.170 & 2.167 & 2.166 & 2.167 & 2.169 & 2.171 & 2.172 \\
\hline & 200 & 1.880 & 1.882 & 1.881 & 1.880 & 1.879 & 1.879 & 1.881 & 1.881 & 1.882 \\
\hline & 220 & 1.327 & 1.330 & 1.329 & 1.328 & 1.327 & 1.327 & 1.328 & 1.329 & 1.331 \\
\hline & 311 & 1.133 & 1.135 & 1.134 & 1.133 & 1.132 & 1.133 & 1.134 & 1.135 & 1.135 \\
\hline & 222 & 1.084 & 1.085 & 1.085 & 1.084 & 1.083 & 1.083 & 1.084 & 1.085 & 1.085 \\
\hline & \multirow[t]{2}{*}{$h k l$} & \multicolumn{9}{|c|}{ Position $(\mu \mathrm{m})$} \\
\hline & & -254 & -200 & -120 & -40 & 0 & 40 & 120 & 200 & 281 \\
\hline \multirow{5}{*}{$5.9 \mathrm{GPa}$} & 111 & 2.171 & 2.169 & 2.163 & 2.153 & 2.148 & 2.153 & 2.616 & 2.168 & 2.171 \\
\hline & 200 & 1.882 & 1.880 & 1.876 & 1.867 & 1.862 & 1.867 & 1.875 & 1.879 & 1.882 \\
\hline & 220 & 1.329 & 1.328 & 1.324 & 1.319 & 1.316 & 1.318 & 1.323 & 1.327 & 1.328 \\
\hline & 311 & 1.135 & 1.133 & 1.131 & 1.126 & 1.123 & 1.126 & 1.130 & 1.133 & 1.134 \\
\hline & 222 & 1.085 & 1.084 & 1.081 & 1.077 & 1.074 & 1.076 & 1.080 & 1.084 & 1.085 \\
\hline
\end{tabular}

angle-dispersive methods on filings of 70-30 $\alpha$-brass (which yielded a value of 1/42). ${ }^{8}$ The FWHM, Gaussian, and Lorentzian integral widths and the Lorentzian fraction increase strongly on filing (Table VI).

The volume compression is illustrated in Fig. 8 for the (111) and (200) lattice spacings with the reference volumes $V_{0}(h k l)$ taken to be those of the undeformed foil (see also Table V). The difference in volume calculated from the (200) and the other reflections remains constant under pressure: The compression behavior is isotropic. The compression curve shows a constant (average) volume between ambient conditions and around $0.3 \mathrm{GPa}$. At pressures above the discontinuity in the compressibility between 0.3 and $0.8 \mathrm{GPa}$, the compression curve is smooth to the highest pressure investigated. The residual compressive stress of $0.3 \mathrm{GPa}$ generated by filing appears to relax slightly under pressure but not on pressure release (Fig. 9). There is no change with pressure in the Voigt fit parameters of any reflection and hence these data are not plotted (selected data are listed in

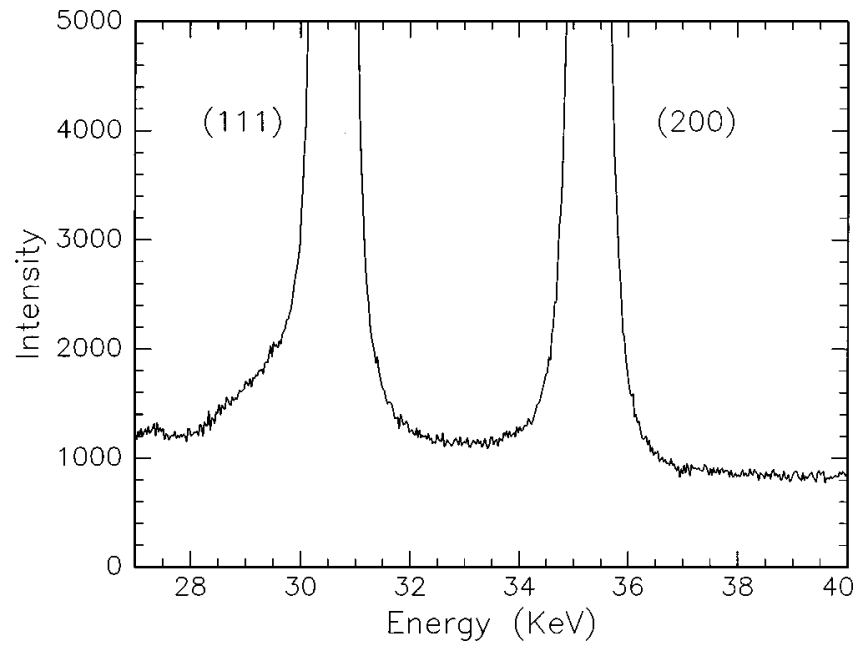

FIG. 7. Energy-dispersive x-ray-diffraction spectrum of the (111) and (200) reflections of $\mathrm{Cu}_{3} \mathrm{Au}$ at $1.3 \mathrm{GPa}$ on pressure reduction. Note the low-energy tail in the (111) reflection. The peak intensity is 76100 counts.
Table VI). Peak shapes were symmetrical with a weak lowenergy tailing present in (111) throughout the experiment and in (200) between 0.3 and $1.3 \mathrm{GPa}$. The size and strain values calculated from the Lorentzian and Gaussian integral widths are presented in Table III. Both the effective and the true size in the $\langle 111\rangle$ direction are very much larger than in the other directions before filing. After filing, the effective and true sizes are more isotropic. Filing seems to have the opposite effect on the strain: Whereas it is isotropic before the experiment, it is markedly anisotropic after the experiment. The largest strains are observed in the $\langle 200\rangle$ direction.

\section{DISCUSSION}

The increase in the FWHM and stress by directly compressing the foil to only $0.3 \mathrm{GPa}$ shows that the lattice is immediately strained. The volume expansion starting between 0.3 and $0.7 \mathrm{GPa}$ and continuing up to $1 \mathrm{GPa}$ cannot be due to tensile stresses (the stresses calculated from the line shifts are compressive, Fig. 2). Since the volume expansion is shown by all directions in the lattice observed, it cannot be due to stacking faults (Fig. 3). Introduction of stacking faults

TABLE V. $d$-spacings as a function of pressure for compression of $\mathrm{Cu}_{3} \mathrm{Au}$ filings in paraffin oil. The second row of values is for the foil after filing. Pressure determined from ruby fluorescence.

\begin{tabular}{cccccc}
\hline \hline $\begin{array}{c}h k l \\
\text { Pressure }(\mathrm{GPa})\end{array}$ & 111 & 200 & $\begin{array}{c}220 \\
\text { spacings }(\AA)\end{array}$ & 311 & 222 \\
\hline 0.0001 & 2.170 & 1.879 & 1.329 & 1.133 & 1.085 \\
& & & & & \\
0.0001 & 2.169 & 1.882 & 1.329 & 1.134 & 1.085 \\
0.33 & 2.169 & 1.882 & 1.329 & 1.134 & 1.085 \\
0.80 & 2.167 & 1.880 & 1.328 & 1.133 & 1.084 \\
1.35 & 2.164 & 1.878 & 1.326 & 1.131 & 1.083 \\
1.80 & 2.162 & 1.875 & 1.324 & 1.130 & 1.081 \\
2.75 & 2.158 & 1.872 & 1.322 & 1.128 & 1.079 \\
4.80 & 2.148 & 1.864 & 1.316 & 1.123 & 1.074 \\
0.0001 & 2.169 & 1.882 & 1.329 & 1.134 & 1.085 \\
\hline \hline
\end{tabular}


TABLE VI. Voigt fit parameters, FWHM, Gaussian $\left(\beta_{G}\right)$, and Lorentzian $\left(\beta_{C}\right)$ integral widths and Lorentzian fraction $\left(\beta_{C} / \beta\right)$ for $\mathrm{Cu}_{3} \mathrm{Au}$ before and after filing, and at $4.8 \mathrm{GPa}$. Data for the FWHM and integral widths are in $\mathrm{eV}$. No data are shown for $\beta_{G}, \beta_{C}$, and $\beta_{C} / \beta$ of the (311) reflection before filing because of overlap with the tungsten fluorescence (from the collimators and slits).

\begin{tabular}{lcccccccccccc}
\hline \hline$h k l$ & \multicolumn{4}{c}{ Before filing } & \multicolumn{4}{c}{ After filing } & \multicolumn{4}{c}{ At 4.8 GPa } \\
& FWHM & $\beta_{G}$ & $\beta_{C}$ & $\beta_{C} / \beta$ & FWHM & $\beta_{G}$ & $\beta_{C}$ & $\beta_{C} / \beta$ & FWHM & $\beta_{G}$ & $\beta_{C}$ & $\beta_{C} / \beta$ \\
\hline 111 & 308 & 126 & 20 & 0.15 & 489 & 203 & 392 & 0.82 & 494 & 202 & 403 & 0.83 \\
200 & 359 & 176 & 67 & 0.31 & 778 & 426 & 708 & 0.78 & 853 & 503 & 750 & 0.75 \\
220 & 417 & 214 & 81 & 0.31 & 803 & 391 & 763 & 0.82 & 814 & 358 & 829 & 0.86 \\
311 & 472 & & & & 1064 & 655 & 900 & 0.72 & 992 & 419 & 1097 & 0.89 \\
\hline \hline
\end{tabular}

on $\{111\}$ planes leads to a lattice contraction as measured by the $\{111\}$ and $\{220\}$ spacings and to a lattice expansion as measured by the $\{200\},\{311\}$, and $\{222\}$ spacings. ${ }^{8,2}$ The volume expansion is attributed here to the generation of dislocations. The stacking faults can then be explained as being due to the splitting and motion of perfect dislocations. ${ }^{9}$ Using the approximation given in Ref. 2 , the dislocation density for a volume increase of $0.5-1.0 \%$ is in the range 3-7 $\times 10^{10} \mathrm{~mm}^{-2}$. While (hydrostatic) pressure should inhibit the creation of dislocations, the stress conditions during the initial compression of a thin foil between anvils are characterized by high values of shear to hydrostatic stress. Note that the compressive uniaxial stress calculated from the line shifts at $0.3 \mathrm{GPa}$ is almost $0.1 \mathrm{GPa}$ (Fig. 2). The introduction of dislocations should lead to an observable increase in the widths of the line profiles. A strong increase was, however, observed only in the pressure regime of constant volume (Fig. 5) or above the discontinuity in the compressibility (Fig. 4). The creation of stacking faults (Fig. 3) and increase in stress (Fig. 2) terminate in the pressure region of the discontinuity in the compressibility, while the line profiles continue to change to 2-4 GPa. Note, however, that most of the change in the line profiles has taken place by $1.3 \mathrm{GPa}$. These

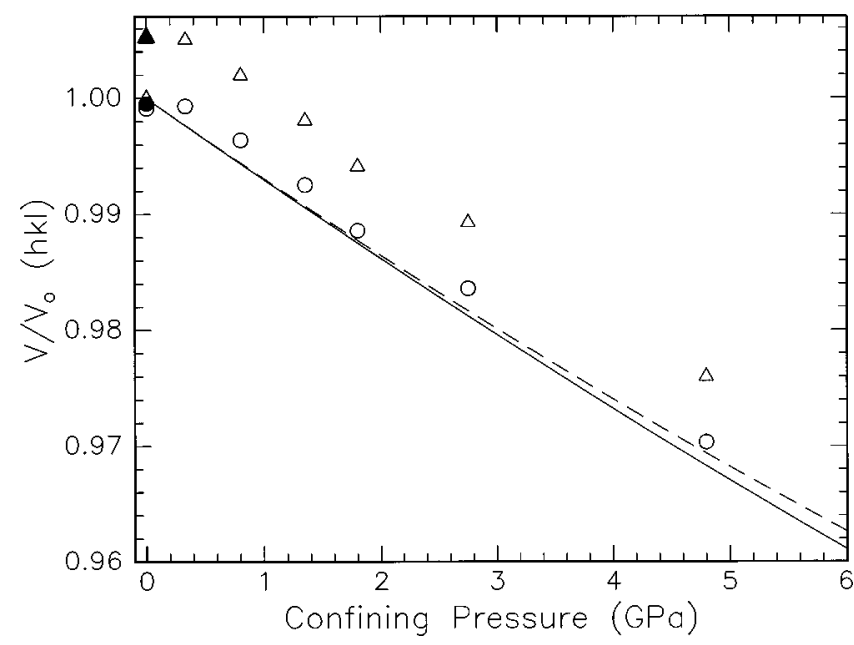

FIG. 8. The scaled volume calculated from the (111, circles) and (200, triangles) lattice spacings for $\mathrm{Cu}_{3} \mathrm{Au}$ filings in paraffin oil vs pressure. The scaled volumes calculated from the (111), (220), and (222) lattice spacings coincide (see Table V). The scaled volumes calculated from the (311) spacing lie in between those calculated from (111) and (200). Solid symbols are for pressure release. Solid and dashed lines as for Fig. 1. results support the interpretation of previous results ${ }^{1,2}$ that the volume incompressibility is due to work hardening. The discontinuity in the compressibility occurs when the yield strength of the material in the plastically deformed state is exceeded such that elastic compression again takes place. The absence of a volume increase and the presence of a volume incompressibility without increase in (residual) stresses in plastically predeformed $\mathrm{Cu}_{3} \mathrm{Au}$ between ambient conditions and $0.3 \mathrm{GPa}$ (for filings) or 1-1.3 GPa [for a foil compressed in a second pressure cycle without pressure medium and in $\mathrm{NaCl}$ (Ref. 1)] shows that there must be an additional physical reason for the incompressibility. This is suggested to be the mutual (elastic) repulsion of defects introduced during plastic deformation which prevents a lattice compression.

The radial scans across the foil at 2.2 and $5.9 \mathrm{GPa}$ show that the foil everywhere is in a fully plastically deformed state. Together with the compression data as a function of pressure at the center of the anvils, they demonstrate that an inner plastically deformed region is being compressed elastically and contained by an outer plastically deformed one (once the yield point at the center has been exceeded). The boundary between the two regions is more than $200 \mu \mathrm{m}$ away from the center. The above general observations are expected on theoretical grounds. ${ }^{10}$

The trends in the compression curves of $\mathrm{Cu}_{3} \mathrm{Au}$ foil in various pressure media [as represented by a scaled average volume, Figs. 10(a), 10(b), and 11] are the same. A pressure

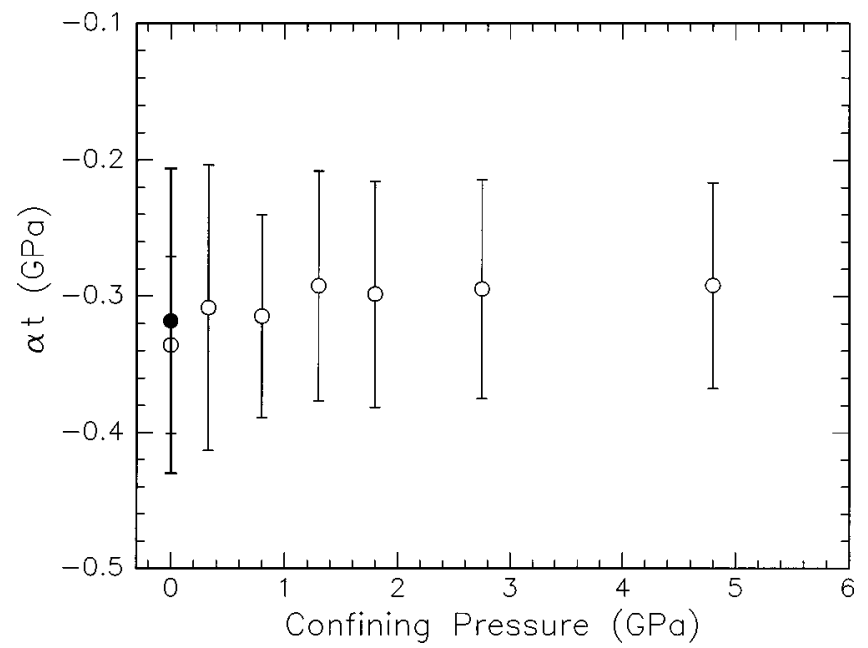

FIG. 9. The product $\alpha t$ vs pressure for $\mathrm{Cu}_{3} \mathrm{Au}$ filings in paraffin oil. 

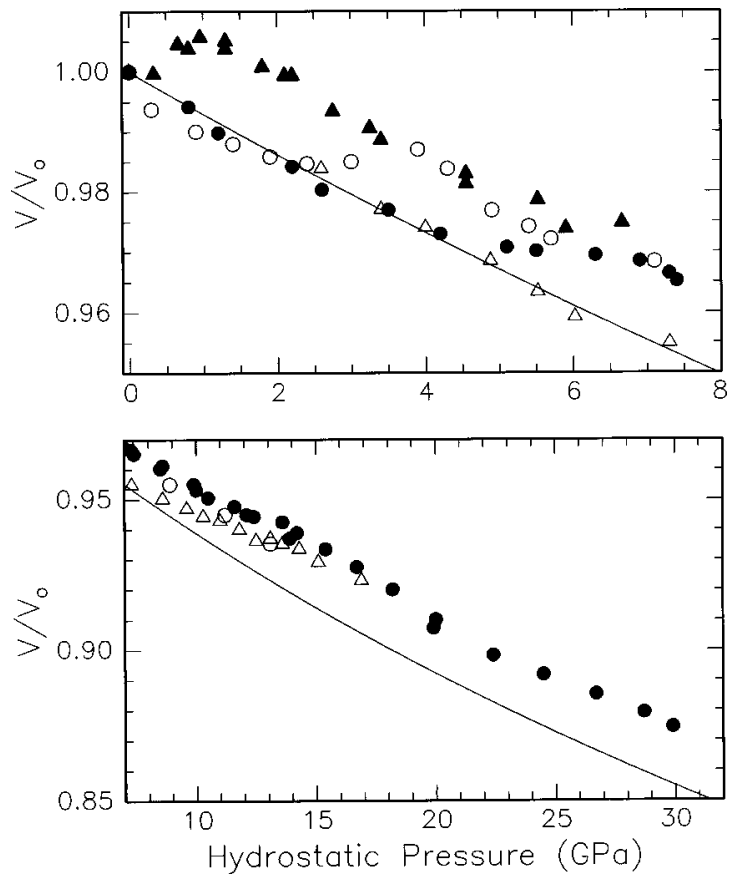

FIG. 10. Compression curves as represented by the scaled average volume for $\mathrm{Cu}_{3} \mathrm{Au}$ in $\mathrm{NaCl}$ (open circles) (Ref. 1), paraffin oil (solid circles), and 4:1 methanol-ethanol (open triangles) (Ref. 2) and for direct compression between diamond anvils (solid triangles).

medium with higher shear strength shifts the beginning of work hardening to lower pressure and extends its pressure range [Figs. 10(a) and 10(b)]. This effect can also be seen in the compression data of $\mathrm{UGa}_{3}$ in silicone oil and methanol-ethanol-water. ${ }^{11}$ The initiation of work hardening thus occurs at a particular ratio of shear to hydrostatic stress and is only coincidentally related to the glass transition in paraffin oil and 4:1 methanol-ethanol. ${ }^{2}$

Above the discontinuity in the compressibility, the volume compression is isotropic, independent of the presence or type of pressure medium and of the initial state of the sample. The present data suggest that the compressibility above the volume discontinuity is similar to the hydrostatic compressibility of the undeformed material. The data of the direct compression of the foil between diamond anvils and of the compression in $\mathrm{NaCl}$ show a constant offset of $1.9 \pm 0.2-0.5 \mathrm{GPa}$ to the hydrostat. The compression data in oil and in methanol-ethanol suggest an increase with pressure in the offset from around $2 \mathrm{GPa}$ in the neighborhood of the discontinuity. The experimental problems during the runs with oil (see Ref. 2) and the lack of data with methanolethanol at high pressures do not allow a firm conclusion to be reached on this issue.

The constant or almost constant offset may be explained as follows. For an elastically compressed isotropic material, the difference in the pressure at which the same volume would occur in the hydrostatic case and in the case of a uniaxial stress superimposed on a hydrostatic stress is ${ }^{12}$

$$
P^{H}-P^{M}=(K / 2 \mu) t
$$

with an additional geometrical factor to account for the specific experimental geometry. ${ }^{1} P^{H}$ and $P^{M}$ are the pressures

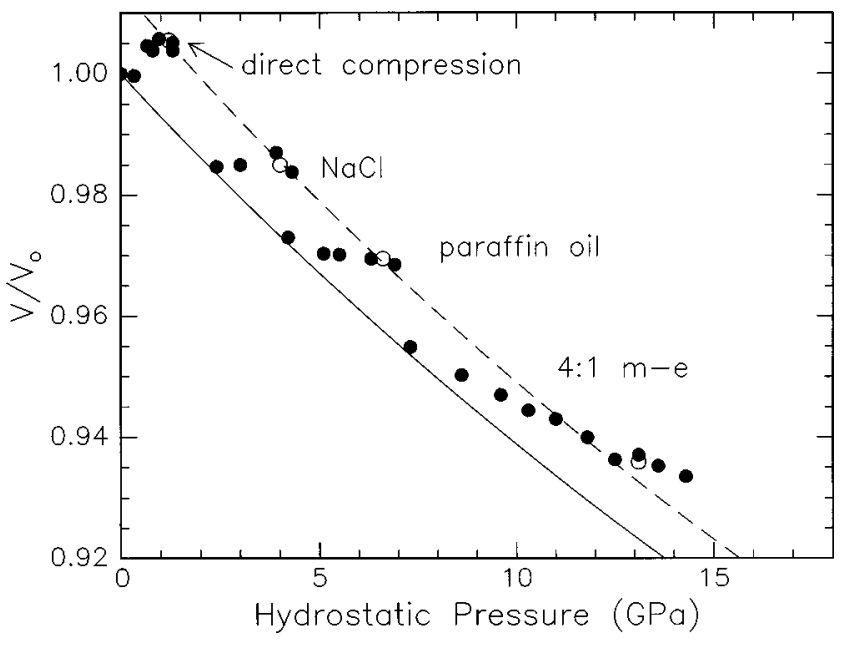

FIG. 11. Hydrostat (solid line) and a curve (dashed line) linking the yield points of $\mathrm{Cu}_{3} \mathrm{Au}$ (shown by open circles) determined in static compression experiments. The loci of the yield points were determined graphically and the curve linking them was fitted with the equation of state of undeformed $\mathrm{Cu}_{3} \mathrm{Au}$ determined in $4: 1$ methanol-ethanol to $7 \mathrm{GPa}$ (Ref. 2) using a constant offset of 1.9 GPa to the hydrostat. The solid circles represent the scaled averaged volume data of $\mathrm{Cu}_{3} \mathrm{Au}$ in different pressure media between the hydrostat and the curve linking the yield points. The horizontal axis represents the pressures measured from ruby. These measured pressures should be close to truly hydrostatic values.

on the hydrostat and under nonhydrostatic conditions, respectively, for the same volume. The difference between them increases with rising uniaxial stress component $t$, depending on the ratio of the bulk modulus $(K)$, to half the shear modulus $(\mu)$. When the stresses saturate at the yield point of the material, the uniaxial stress component $t=\left(\sigma_{3}\right.$ $-\sigma_{1}$ ) is equal to the yield strength $Y$ according to the von Mises yield criterion. If the compression continues to have a hydrostatic component above the yield point, the offset in pressure between the measured compression curve and the true hydrostat should depend on pressure only through the ratio $K / 2 \mu$. If this ratio does not change much, the offset above the discontinuity in the compressibility (the yield point in the plastically deformed state) will be constant and a measure of the yield strength of the plastically deformed material.

The arguments just presented are analogous to those used in explaining the Hugoniot compression curves in shock wave experiments. ${ }^{13-15}$ In these experiments, the compression is purely uniaxial $\left[\sigma_{3}=(2 \mu+\lambda)(\Delta V / V), \sigma_{2}=\sigma_{1}\right.$ $=\lambda(\Delta V / V)$, where $\lambda$ is the Lamé coefficient]. The offset between the hydrostat and the Hugoniot is then -(4/3) $\mu(\Delta V / V)$ which, again by the von Mises yield criterion, becomes (2/3) $Y$ at the yield point. Above the yield point on the Hugoniot (called the Hugoniot elastic limit), the offset is constant at this value if the yield stress does not increase with pressure. ${ }^{14}$

The yield points in the present static compression experiments with and without pressure media lie on a single curve with a constant or almost constant offset of $1.9 \pm 0.2-0.5$ GPa to the hydrostat [Figs. 10(a), 10(b), and 11]. This suggests that the compression curve above the yield point represents uniaxial compression. The yield strength of $\mathrm{Cu}_{3} \mathrm{Au}$ 
deformed in the static experiments is thus estimated to be 2.9 $\mathrm{GPa}$. This is far above the value which would be calculated from $\alpha t$ (where $t=\sigma_{3}-\sigma_{1}=Y$ ) which is between 0.1 and $0.4 \mathrm{GPa}$. A value of $2.9 \mathrm{GPa}$ would be expected from a crystallite size of about $14 \mathrm{~nm}$ which is in the range calculated from the Lorentzian integral widths [Table V; it is not clear, however, that the Hall-Petch relation found for $\mathrm{Cu}_{3} \mathrm{Au}$ (Ref. 16) can be extrapolated to such small crystallite sizes]. A similar discrepancy between $t$ and $Y$ determined by somewhat different $\mathrm{X}$-ray techniques (albeit also based on considerations of linear elasticity) for $\mathrm{W}$ and $\mathrm{Fe}$ in high-pressure studies has recently been noted. ${ }^{17}$

The discontinuities of the compression curves in the static and dynamic compression experiments have the same physical origin. From this point of view, it is interesting to compare the defect structures of disordered $\mathrm{Cu}_{3} \mathrm{Au}$ in the static and dynamic experiments. The stacking fault probabilities of the foil at high pressures and of the filings at ambient conditions agree well with that determined for disordered $\mathrm{Cu}_{3} \mathrm{Au}$ shocked to $16 \mathrm{GPa}(0.0023){ }^{18}$ The maximum residual compressive stress in that study $(0.37 \mathrm{GPa}$, assuming the fraction of the Reuss state to be $\alpha=0.5$ ) occurred at a shock pressure of $29 \mathrm{GPa}$. The true crystallite sizes of the filings are close to those of the sample shocked to $47.5 \mathrm{GPa}$ (where the stacking fault probability is much higher than for the filings). The trends in crystallite size with $h k l$ agree for the directly compressed foil, the filings, and the shocked samples.

A correction of $[(2 / 3) Y]$ was discussed for the static com- pression experiments establishing the secondary ruby pressure scale above $6 \mathrm{GPa}^{4}$ The experiments with direct compression of foils and with compression of powders without pressure medium in that work can have sampled the compression curves only above the yield points of the materials (note that the elastic and mechanical properties of $\mathrm{Cu}_{3} \mathrm{Au}$ are very close to those of $\mathrm{Cu}$; the yield point for $\mathrm{Cu}$ is therefore expected to occur around $1.5 \mathrm{GPa}$ in compression experiments without pressure medium or gasket). The corrections were not applied because they were not determined experimentally in the static experiments, because of the uncertain effects of temperature in the dynamic experiments and because the corrections of $[(2 / 3) Y]$ were proposed to cancel in the dynamic and static high-pressure experiments. ${ }^{4}$

The present work is the first experimental demonstration, to our knowledge, of the kind of corrections that need to be applied in nonhydrostatic experiments above the yield point. In experiments with ill-defined starting materials and states of nonhydrostatic stress, compression curves between the extremes of direct compression (Fig. 1) and the hydrostat will be obtained provided that pressure is measured correctly and independently. The data would be interpreted as exhibiting scatter about a hydrostat if large pressure steps were taken while they actually lie on well-defined compression curves. The present experiments show that hydrostatic compression experiments on powders require not only a hydrostatic pressure medium but also a powder consisting essentially of single crystals. Heating of polycrystals between pressure steps may not anneal the strains and defects but rather induce new ones because of thermal anisotropy.
${ }^{1}$ J. W. Otto, J. K. Vassiliou, and G. Frommeyer, J. Synchrotron Radiat. 4, 155 (1997).

${ }^{2}$ J. W. Otto, J. K. Vassiliou, and G. Frommeyer, preceding paper Phys. Rev. B 57, 3253 (1998).

${ }^{3}$ R. A. Forman, G. J. Piermarini, J. D. Barnett, and S. Block, Science 176, 284 (1972).

${ }^{4}$ H. K. Mao, P. M. Bell, J. W. Shaner, and D. J. Steinberg, J. Appl. Phys. 49, 3276 (1978).

${ }^{5}$ J. W. Otto, Nucl. Instrum. Methods Phys. Res. A 384, 552 (1997).

${ }^{6}$ J. W. Otto, J. Appl. Crystallogr. (to be published).

${ }^{7}$ M. Yamaguchi and Y. Umakoshi, Prog. Mater. Sci. 34, 1 (1990).

${ }^{8}$ B. E. Warren, X-ray Diffraction (Dover, New York, 1990).

${ }^{9}$ D. Hull and D. J. Bacon, Introduction to Dislocations, 3rd ed. (Pergamon Press, Oxford, 1984).

${ }^{10} \mathrm{~J}$. W. Jackson and M. Waxman, in High Pressure Measurement, edited by A. A. Giardini and E. C. Lloyd (Butterworth, Washington, D.C., 1963).
${ }^{11}$ T. LeBihan, S. Heathman, S. Darracq, C. Abraham, J-M. Winand, and U. Benedict, High Temp.-High Press. 27/28, 157 (1995/ 1996).

${ }^{12}$ Y. Meng, D. J. Weidner, and Y. Fei, Geophys. Res. Lett. 20, 1147 (1993).

${ }^{13}$ G. R. Fowles, J. Appl. Phys. 32, 1475 (1961).

${ }^{14}$ M. H. Rice, R. G. McQueen, and J. M. Walsh, in Solid State Physics, edited by F. Seitz and D. Turnbull (Academic Press, New York, 1958), Vol. 6.

${ }^{15}$ O. E. Jones and R. A. Graham, in Accurate Characterization of the High Pressure Environment, Natl. Bur. Stand. Spec. Publ. No. 326, edited by E. C. Lloyd (U.S. GPO, Washington, D.C., 1971).

${ }^{16}$ S. M. L. Sastry, Mater. Sci. Eng. 33, 237 (1976).

${ }^{17}$ R. J. Hemley, H.-K. Mao, G. Shen, J. Badro, P. Gillet, M. Hanfland, and D. Häusermann, Science 276, 1242 (1997).

${ }^{18}$ D. E. Mikkola and J. B. Cohen, Acta Metall. 14, 105 (1966). 